Petra Kirberger

\title{
Registrar und Genossenschaftsregisterrichter
}

Rechtsstellung und Aufgabenbereich nach englischem, tansanischem und deutschem Recht Marburger Schriften zum Genossenschaftswesen Reihe B/Band 14

Veröffentlichungen des Instituts für Kooperation in Entwicklungsländern der Philipps-Universität Marburg/Lahn herausgegeben von Prof. Dr. Eberhard Dülfer, Göttingen 1977, XVII, 164 S.

Trotz ihrer auf den ersten Blick recht speziellen Themenstellung verdient diese rechtsvergleichende Dissertation hier Erwähnung. Die Autorin geht von dem klassischen Erkenntnisinteresse jeder Rechtsvergleichung, nämlich dem Streben nach Rechtsvereinheitlichung aus, das sie allerdings von vornherein auf die beiden untersuchten EG-Staaten, Großbritannien und die Bundesrepublik, beschränkt (S. 1). Dennoch werden die genossenschaftsrechtlichen Regelungen einiger afrikanischer Commonwealth-Staaten (Kenia, Tansania, Ghana, Uganda u. Sambia) in die Untersuchung einbezogen, wobei das tansanische Recht als Beispiel für die afrikanische Entwicklung ausführlich dargestellt wird (S. 54 ff.).

Die Bedeutung einer solchen Untersuchung für das Verständnis einer Entwicklungsstrategie, in der die Bildung und Kontrolle von Genossenschaften als Säulen der (community-ba-) sed society eine wichtige Rolle spielen, liegt auf der Hand. Allerdings werden auch schnell die Grenzen eines Rechtsvergleichs deutlich, der sich zudem auf die gesetzlichen Vorschriften beschränkt: Nachdem die Autorin nacheinander die englische, die afrikanische und die deutsche Rechtslage dargestellt hat, kann sie nicht umhin, die Inkommensurabilität der afrikanischen und der europäischen Regelungen festzustellen (S. 133 ff.) und den eigentlichen Rechtsvergleich auf letztere zu beschränken, denn das Genossenschaftsrecht hat - wie knapp, aber zutreffend herausgearbeitet wird - in einem Staat der Dritten Welt eine völlig andere ökonomische Funktion als in Europa; der afrikanische Registerrichter hat dementsprechend viel weitergehende Einwirkungsmöglichkeiten als sein europäischerKollege, die er zur Verwirklichung bestimmter wirtschaftspolitischer Ziele zu nutzen hat. Über die Rolle und Wirkung des Genossenschaftsrechts im Entwicklungsprozeß z. B. Tansanias will und kann diese normenvergleichende Arbeit dagegen keinen Aufschluß geben.

Alexander Dix

LesLIE T. C. KuO

\section{Agriculture in the People's Republic of China}

Structural Change and Technical Transformation

Praeger Special Studies in International Economics and Development, Praeger Publishers, New York, Washington, London, 1976, XX, 288 S., \$23.50

Die Entwicklung der Landwirtschaft in der Volksrepublik China ist von großer Bedeutung für das Verständnis der chinesischen Gegenwart insgesamt wie auch für die vergleichende Betrachtung des Agrarsektors in anderen asiatischen Ländern. Ob das chinesische Modell Vorbild für andere Staaten und Regionen sein kann, wird sich vor allem auf dem Agrarsektor erweisen müssen. Dem Gegenstand wissenschaftlich gerecht zu werden, ist schwierig wegen der nur in äußerst begrenztem Umfang gegebenen Möglichkeiten, sich vor Ort ein Urteil zu bilden. Die Beurteilung muß sich deshalb weitgehend auf Daten stützen, deren Zuverlässigkeit in Frage steht, weil ihre Ubermittler an bestimmten Schlußfolgerungen interessiert sein können - dies gilt für offizielles chinesisches Material wie für solches, das aus Institutionen in Hongkong oder Taiwan stammt. Intime Kenntnis der Verhältnisse, Zugang zu den vielfälti- 